
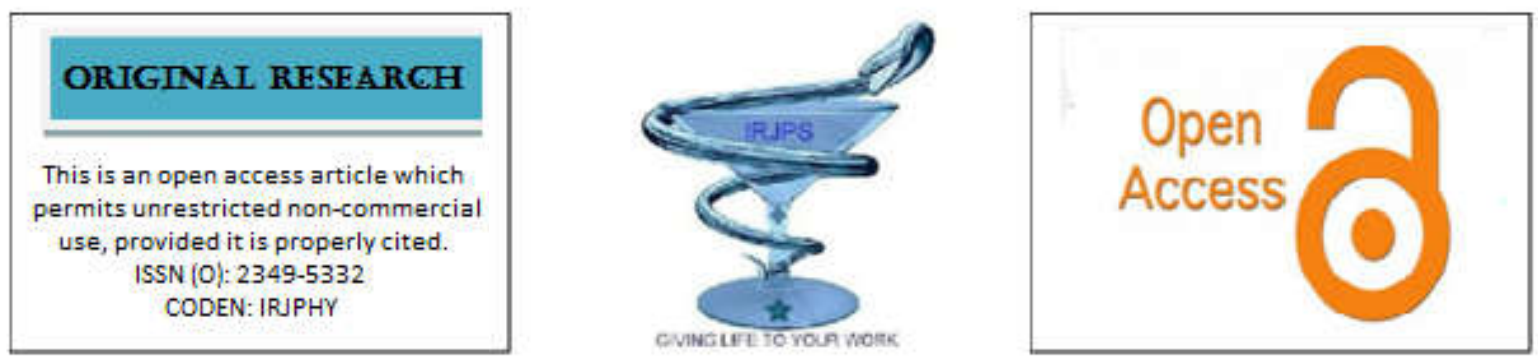

\title{
COMPARATIVE IN VITRO DISSOLUTION STUDY OF A NOVEL ACECLOFENAC AND PARACETAMOL FIXED DOSE COMBINATION WITH MARKETED FORMULATION IN INDIA
}

\author{
Sunil Agarwal ${ }^{1}$, Sambhaji Deshmukh ${ }^{1}$, Hiten Saresa ${ }^{2 *}$, Kapil Dev Mehta ${ }^{2}$, Rishi Jain ${ }^{2}$ \\ ${ }^{1}$ Pharma Research,Wockhardt Research Centre,Aurangabad, India \\ ${ }^{2}$ Department of Medical Affairs, Wockhardt Ltd, BKC, Mumbai, India
}

Submitted on: 30.03.19;

Revised on: 22.04.19;

Accepted on: 13.05.19

\begin{abstract}
:
Introduction: Aceclofenac has low aqueous solubility leading to poor dissolution and insufficient oral bioavailability. The improvement of its dissolution is an important issue for enhancing its onset of action and therapeutic efficacy.

Aim: To compare in vitro dissolution of a novel aceclofenac and paracetamol fixed dose combination with reference product available in India.

Methods: The new formulation consists of paracetamol and aceclofenac (Ace-Proxyvon) as the active ingredients blended in a readily dispersible matrix with a combination of Effer-soda ${ }^{\circledR}$ and novel combination of buffer salts. This new formulation was compared with the reference brand available in Indian market. A comparative dissolution study was performed in $\mathrm{pH} 4.5$ acetate buffer. Peak area of Ace-Proxyvon and reference product were measured and recorded on High Performance Liquid Chromatography system.

Results: The invitro release data (cumulative percent drug release) have been plotted against time to get a graphical presentation of the data. All dissolution data are based on the actual drug content of the test tablets as calculated from the assay results. Around $64 \%$ of aceclofenac and $88 \%$ of paracetamol were released within 15 min from AceProxyvon as compared to $32 \%$ of aceclofenac and $79 \%$ of paracetamol from reference product in $500 \mathrm{ml}, \mathrm{pH} 4.5$ acetate buffer.

Conclusion: In this study, aceclofenac showed faster dissolution as compared to reference product in $500 \mathrm{ml}, \mathrm{pH} 4.5$ acetate buffer. The addition of novel excipients like Effer-soda ${ }^{\circledR}$ and combination of buffer salts can lead to faster onset and better therapeutic efficacy of Ace-Proxyvon as compared to reference product.
\end{abstract}

KEYWORDS: Solid dosage form, Aceclofenac, Paracetamol, invitro dissolution, onset of action

Corresponding Author: Hiten Saresa

E mail: hsaresa@wockhardt.com

Mobile: 9825760315
Indian Research Journal of Pharmacy and Science; 20(2019) 1825-1831;
Journal Home Page: https://www.irjps.in
DOI: 10.21276/irjps.2019.6.1.8 


\section{INTRODUCTION:}

Drug dissolution testing is an analytical technique used to assess release profiles of drugs from pharmaceutical products, generally solid oral products such as tablets and capsules ${ }^{1}$. If a drug from its dosage form has to produce its effect, it must be released from the product and should generally be dissolved in the fluids of the gastrointestinal tract. The main purpose of solid dosage form is to make a drug available to the human body at a certain rate and define amount through the GIT so that the drug can produce pharmacological effects. But studies on bioavailability of drugs from a given dosage form revealed that, in many situations, solid dosage forms did not give the same therapeutic effects. This is mainly due to the insufficient dissolution and subsequent absorption of the drug from the GIT. So, dissolution analysis of pharmaceutical solid dosage forms is a very important test of product quality ${ }^{2}$.

Aceclofenac (2-[(2, 6-diclorophenyl) amine] phenyl acetoxyacetic acid) is a non-steroidal antiinflammatory drug (NSAID) of phenyl acetic acid group, which possesses remarkable antiinflammatory, analgesic and anti-pyretic properties ${ }^{3,4}$. It is widely used for the relief of pain and inflammation in rheumatoid arthritis, osteoarthritis and ankylosing spondylitis. The dose is $100 \mathrm{mg}$ twice daily. Aceclofenac shows comparatively higher antiinflammatory action than conventional NSAIDs ${ }^{5,6,7}$.The drug works by blocking the action of cyclooxygenase that is involved in the production of prostaglandins causing pain, swelling and inflammation. Aceclofenac appears to be well tolerated among NSAIDs with a lower incidence of gastrointestinal adverse effects ${ }^{8}$. Unfortunately aceclofenac suffers from low aqueous solubility $(0.058 \mu \mathrm{g} / \mathrm{ml})$, leading to poor dissolution and insufficient oral bioavailability. The biopharmaceutical classification system (BCS) divides all drug candidates into four different groups, according to their solubility and permeability ${ }^{9}$. Aceclofenac is an example of BCS class II compound (highly permeable \& low soluble) ${ }^{10,11}$; its oral bioavailability is determined by dissolution rate in the gastrointestinal tract ${ }^{12,13}$. Therefore the improvement of Aceclofenac dissolution is an important issue for enhancing its onset of action and therapeutic efficacy.
Aceclofenac is soluble in the alkaline environment ( $\mathrm{pH}$ 7-7.5) and it is practically insoluble in gastric $\mathrm{pH}$ of $1-1.5^{14}$. Since the drug dissolution in the physiological environment of the GI tract is the primary step in the oral absorption process, only dissolved drug can permeate the mucosa at the absorptive sites in the GI tract ${ }^{15}$. Therefore for Aceclofenac, relief from pain would be unlikely until the drug traverses the intestinal part (pH 6 and above) which takes about 1-2 hours. To provide faster pain relief the dissolution of Aceclofenac in the gastric fluid is of prime importance. With this rationale a new fast disintegrating and rapidly soluble tablet was developed at Wockhardt Research and Development Centre. The formulation consists of paracetamol and aceclofenac as the active ingredients blended in a readily dispersible matrix with a combination of Effer-soda ${ }^{\circledR}$ and superdisintegrants (sodium starch glycolate) and a novel combination of buffer salts to provide a $\mathrm{pH}$ micro-environment suitable enough to increase the solubility of aceclofenac.

To assess the standard of a product, in vitro dissolution test is widely used because, for any solid dosage forms, gastrointestinal absorption first requires dissolution of the tablet or capsule that liberates the drug into solution ${ }^{16}$. The dissolution characteristic of a drug from the dosage form depends on many factors including its formulation and manufacturing process ${ }^{17}$. This study deals with the assessment of comparative dissolution profile of aceclofenac and paracetamol fixed dose combination (FDC)Tablets of M/s Wockhardt (Ace-Proxyvon) \& reference marketed formulation.

\section{MATERIALS AND METHODS:}

Drug: Aceclofenac WS (Amoli Oganics Pvt. Ltd., India), Paracetamol WS (Bharat Chemicals)

Solvents and reagents: Sodium acetate trihydrate (AR Grade) (Merck, India), Acetonitrile (HPLC Grade) (Merck, India), Methanol (HPLC Grade) (Merck, India), Triethylamine (GR Grade) (Merck, India), Acetic acid (GR Grade) (Merck, India), Potassium dihydrogen phosphate (GR Grade) (Merck, India), Sodium hydroxide (GR Grade) (Merck, India), Sodium Acetate Trihydrate (GR Grade) (Merck, India), Acetic Acid (GR Grade) (Merck, India), Water (Milli-Q / HPLC Grade). 
Equipments: HPLC system (Waters Alliance), Digital pH meter (Orion Star A211), Tablet dissolution tester (Electro lab, India), Electronic balance (Metler Toledo)

\section{Dosage forms}

Ace-Proxyvon tablets, manufactured by - Wockhardt Limited was compared against reference aceclofenac and paracetamol fixed dose combination formulation available in the market.

These samples were properly checked for their batch number, manufacturing and expiry dates before the study. The labelled active ingredients were Aceclofenac $100 \mathrm{mg}$ and Paracetamol 325.

\section{In discriminatory and biorelevant dissolution medium}

A discriminatory and biorelevant dissolution system able to simulate the conditions of the human GI tract in terms of dosing conditions and therapeutic objective was selected. Since NSAIDs are recommended to be administered after meals and typical $\mathrm{pH}$ values measured soon after food intake ranges from 4-4.5, a 4.5 acetate buffer medium which reflects the GI conditions that is relevant to drug release from this formulation was selected. Further with respect to the volume of the medium, gastric juice secretion is usually low in fasted state with the result that the volume of fluid available to dissolve the dose is much lower than the standard dissolution test volume of $900 \mathrm{ml}$ or $1000 \mathrm{ml}$. Taking into account the co-ingested fluid and food a more reasonable volume is in the order of 400-500 $\mathrm{ml}$. In this study, dissolution parameter was: Dissolution Medium- 500 ml, pH 4.5 Acetate Buffer, Dissolution Apparatus- USP apparatus II (Paddle) RPM: 50, Temp of dissolution medium: $37 \pm 0.5^{\circ} \mathrm{C}$.

Preparation of dissolution medium: (pH 4.5 Acetate buffer):

$2.9 \mathrm{~g}$ sodium acetate trihydrate was dissolved in 900 $\mathrm{ml}$ water. $14 \mathrm{ml}$ of $2 \mathrm{~N}$ acetic acid was added and diluted to mark $1000 \mathrm{ml}$ with water. If necessary, $\mathrm{pH}$ was adjusted to $4.5 \pm 0.05$ with dilute acetic acid or $2 \mathrm{~N}$ sodium hydroxide.

\section{Preparation of Standard Solution:}

Accurately weighed $28 \mathrm{mg}$ Aceclofenac and $90 \mathrm{mg}$ Paracetamol working standard were transferred to 50 $\mathrm{ml}$ volumetric flask. $5 \mathrm{ml}$ methanol was added to it and sonicated to dissolve and was diluted to the mark with dissolution media and mixed. $5 \mathrm{ml}$ of this primary stock solution was taken in $25 \mathrm{ml}$ volumetric flask and diluted to mark with dissolution media and mixed.

\section{Performance of Dissolution study:}

\section{$500 \mathrm{ml}$, pH 4.5 Acetate Buffer}

$500 \mathrm{ml}$ medium was added to each vessel and waited till media temperature was not achieved to $37 \pm 0.5^{\circ} \mathrm{Cfor}$ each vessel. The apparatus II (paddle) was then assembled and rotation set to $50 \mathrm{rpm}$ and then system was allowed to equilibrate for 15 minutes. Six tablets of each manufacturer were dropped in each vessel and apparatus was immediately operated for 60 minutes for each set of sample. Six time points were selected for dissolution profile at 5, 10, 15, 30, 45, $60 \mathrm{~min}$. $10 \mathrm{ml}$ of aliquot was withdrawn from each timepoint and filtered through $0.45 \mu$ nylon filter. Dissolution medium as injected in HPLC as blank, standard solution (five injections) and sample solution (one injection). Peak area of standard and samples were measured and recorded on HPLC system.

\section{RESULTS}

Drug dissolution profiles may be distinct due to differences in formulations and manufacturing processes, but the differences must not compromise product bioequivalence. The in-vitro release data (cumulative percent drug release) have been plotted against time to get a graphical presentation of the data. All dissolution data are based on the actual drug content of the test tablets as calculated from the assay results. The release profiles of different brands of aceclofenac and paracetamol FDC tablets are shown in Fig. $1 \& 2$ and Table $1(500 \mathrm{ml}, \mathrm{pH} 4.5$ Acetate Buffer). During first 10 mins $47 \%$ of aceclofenac was released from Ace-Proxyvon as compared to $28 \%$ from reference brand. Around $64 \%$ of aceclofenac and $88 \%$ of paracetamol were released within $15 \mathrm{~min}$ from Ace-Proxyvon as compared to $32 \%$ of aceclofenac and $79 \%$ of paracetamol from reference product in $500 \mathrm{ml}, \mathrm{pH} 4.5$ acetate Buffer. At $1 \mathrm{hr}$ around $65 \%$ of aceclofenac and $90 \%$ of paracetamol were released from Ace-Proxyvon as compared to $44 \%$ of aceclofenac and $84 \%$ of paracetamol from reference product. 
Table 1: Comparative Dissolution Study

\begin{tabular}{|c|c|c|c|c|}
\hline & \multicolumn{2}{|c|}{ Dissolution for Aceclofenac } & \multicolumn{2}{|c|}{ Dissolution for Paracetamol } \\
\hline Medium & \multicolumn{2}{|c|}{$\mathrm{pH} 4.5$, Acetate Buffer } & \multicolumn{2}{|c|}{$\mathrm{pH} 4.5$, Acetate Buffer } \\
\hline Apparatus & \multicolumn{2}{|c|}{ Paddle } & \multicolumn{2}{|c|}{ Paddle } \\
\hline Volume & \multicolumn{2}{|l|}{$500 \mathrm{ml}$} & \multicolumn{2}{|l|}{$500 \mathrm{ml}$} \\
\hline RPM & \multicolumn{2}{|l|}{50} & \multicolumn{2}{|l|}{50} \\
\hline Time point & Ace-Proxyvon & Reference brand & Ace-Proxyvon & Reference brand \\
\hline 5 & 24 & 22 & 64 & 66 \\
\hline 10 & 47 & 28 & 85 & 76 \\
\hline 15 & 64 & 32 & 88 & 79 \\
\hline 30 & 64 & 38 & 88 & 82 \\
\hline 45 & 65 & 42 & 88 & 83 \\
\hline 60 & 65 & 44 & 90 & 84 \\
\hline
\end{tabular}

Results in 500 ml, pH 4.5 Acetate Buffer

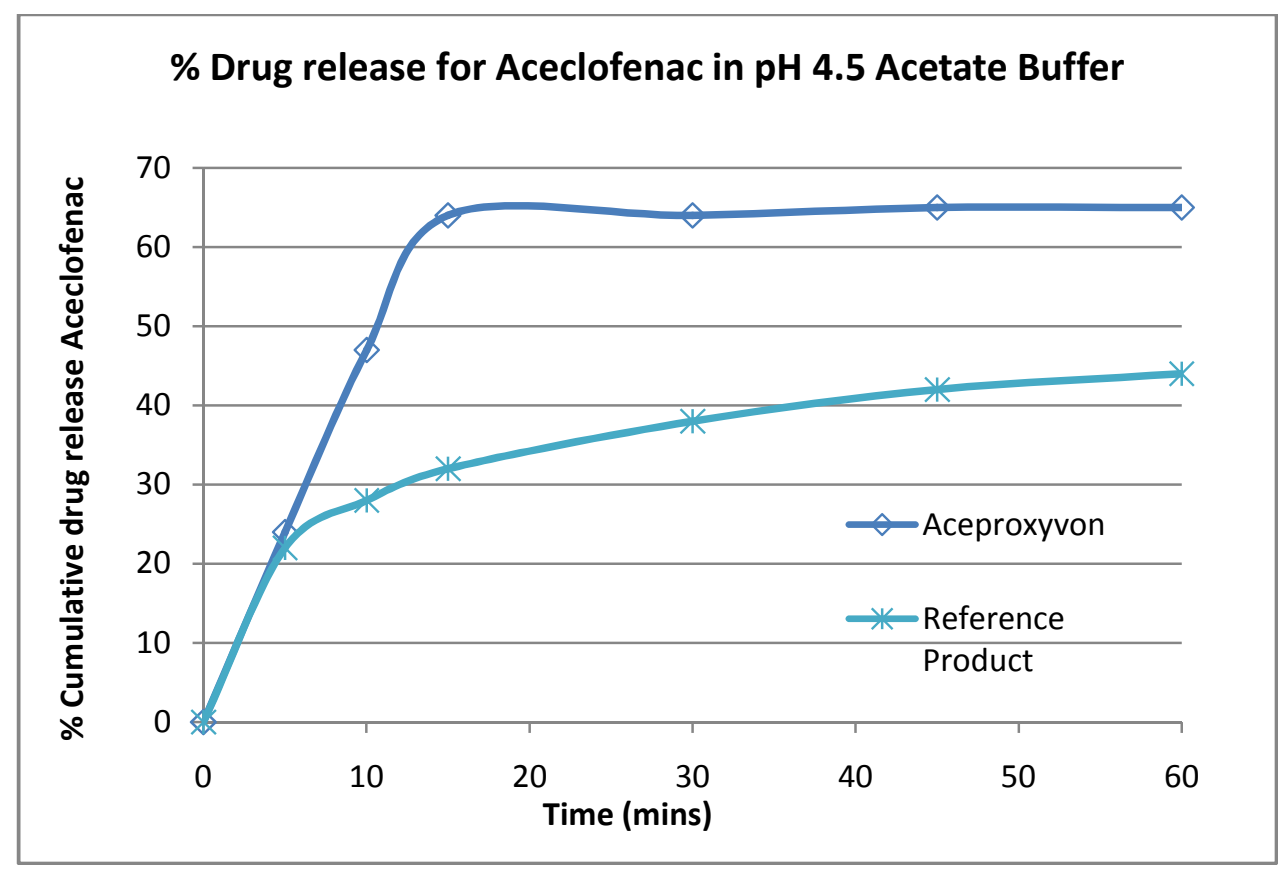

Figure 1: \% Drug release for aceclofenac in pH 4.5 acetate buffer 


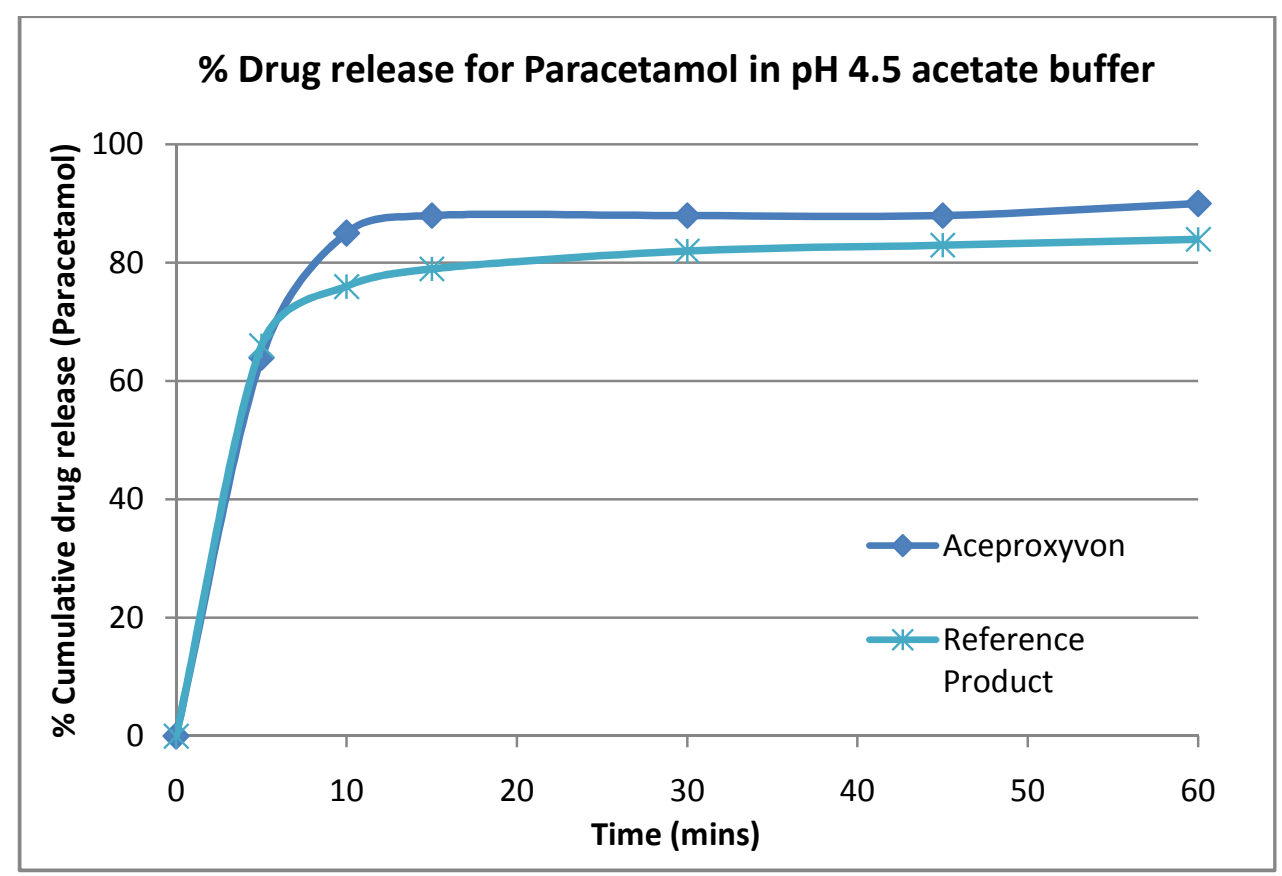

Figure 2:\% Drug release for paracetamol in pH 4.5 acetate buffer

\section{DISCUSSION:}

Drug dissolution testing is a quantitative analytical technique for assessing drug release from pharmaceutical products, in particular solid oral dosage forms such as tablets and capsules ${ }^{18}$. The reason for conducting the test is that generally for a drug to be absorbed, usually from the gastrointestinal tract, the drug should be in solution form. Thus evaluation of dissolution becomes useful and necessary ${ }^{19,20,21}$.Faster dissolution of aceclofenac seen in this study may have been due to the presence of superdisintegrants and some special alkalizing agents in the formulation which increase the $\mathrm{pH}$ of the dissolution media and facilitate drug release. Superdisintegrants like sodium starch glycolate is used as a pharmaceutical grade dissolution excipient for tablets and capsules. Sodium starch glycolate absorbs water rapidly, resulting in swelling which leads to rapid disintegration of tablets. C. Mallikarjuna Setty et al. also demonstrated that fastdispersible aceclofenac tablets could be prepared by direct compression using superdisintegrants ${ }^{22}$.

Effer-Soda ${ }^{\circledR}$ is a highly stable, surface modified sodium bicarbonate powder developed for use in pharmaceutical and nutraceutical applications ${ }^{23}$. Effer-Soda $^{\circledR}$, is a novel excipient comprising of coated sodium bicarbonate which provides stability to the formulation against moisture and helps in creating an alkaline micro-environment for the dissolution enhancement of drugs having solubility in alkaline environment. Aceclofenac is soluble in the alkaline environment ( $\mathrm{pH}$ 7-7.5) and it is practically insoluble in gastric $\mathrm{pH}$ of 1-1.5. Therefore by creating alkaline microenvironment, the increased dissolution of aceclofenac was seen in the $\mathrm{pH} 4.5$, which also mimics the $\mathrm{pH}$ of stomach in fed conditions. Similar results were also reported by Srivastav et al. (2011) in case of gliclazide, a poorly water soluble drug ${ }^{24}$.

\section{CONCLUSION:}

The present study demonstrates that novel aceclofenac and paracetamol fixed dose combination developed by Wockhardt Research Centre shows faster dissolution as compared to reference product. Unique excipient combination of buffer salts, surface modified sodium bicarbonate and superdisintegrants have resulted in increased solubility \& faster dissolution of aceclofenac. This enhancement in dissolution rate may further result in rapid onset of action and better therapeutic efficacy. 


\section{REFERENCES:}

1.USP General Chapter on Dissolution . United States Pharmacopoeia and National Formulary; United States Pharmacopoeial Convention, Inc.: Rockville, MD, 2005; pp 2412-2414.

2. U.S. FDA/CDER, Guidance for Industry, "Dissolution testing of immediate release solid oral dosage forms", 1997.

3. British pharmacopoeia, The Stationary office, MHRA, volume 1, British PharmacopoeialCommission office, London, 2005.

4. K. Parfitt, Analgesics anti-inflammatory and antipyretics, in: J.E.F. Reynolds (Ed.), Martindale: The Complete Drug Reference. 32nd ed., 1999, pp. 2-12, Massachusetts.

5.Perez-Ruiz F, Alonso-Ruiz A, Ansoleaga JJ. Comparative study of the efficacy and safety of aceclofenac and tenoxicam in rheumatoid arthritis.ClinRheumatol. 1996;15(5):473-7.

6.Martín-Mola E, Gijón-Baños J, AnsoleagaJJ..Aceclofenac in comparison to ketoprofen in the treatment of rheumatoid arthritis.Rheumatol Int. 1995;15(3):111-6.

7. Pasero G, Marcolongo R, Serni U, Parnham MJ, Ferrer F.A multi-centre, double-blind comparative study of the efficacy and safety of aceclofenac and diclofenac in the treatment of rheumatoid arthritis. Curr Med Res Opin. 1995;13(6):305-15.

8. K. V. Gowda, D. S. Rajan, U. Mandal, P. S. Selvan, W. D. Sam Solomon, A. Bose, A. K. Sarkar, T. K. Pal, T. K. Chattaraj, Evaluation of bioequivalence of two formulations containing $100 \mathrm{mg}$ of Aceclofenac, Drug Dev, Ind. Pharm, 32(2006) 19-25.

9. Amidon L Gorden, Raimer Loebenberga, Modern bioavilability, bioequivalence international regulatory standards, Eur. J. Pharm . Biopharm 50 (2000) 3-12.

10. Ahmad Z, Maurya N, Mishra KS, Khan I. Solubility enhancement of poorly water soluble drugs: a review. International Journal of Pharmacy \& Technology 2011; 3(1): 807-823. 5. USP-NF. The Official Compendia of Standards. Reference Table/Description And Solubility, USP, 2006; 29: 3191

11. Wankhedenaveet B, Walekr S. S, Sadgir P. S, Pawar S. A, Ahirrao S. P, liquid solid A Novel technique for dissolution enhancement of poorly water soluble drugs, Asian Journal of Pharmaceutical Technology and Innovation, 2014; 02(08): 77-90.

12. B. Lee, H. Jung, Enhanced bioavailability of poorly water -soluble Aceclofenac using PEG-based Solid dispersion in rats, beagle dogs and human subjects, AAPS Annual Meeting, New Orleans, LA, USA, Pharm. Sci. suppl., 4, 1999 , pp. 14-18.

13. T. Kim, J. Shin, B. Lee, Enhanced Dissolution and bioavailability of poorly-water soluble Aceclofenac using solid dispersion system, Denver, Colarado, USA:AAPS ,Annual Meeting, 2001 ,pp.21-25.

14. Islam, Ashraf \& Islam, Sharmi\&Shahriar, Mohammad \&Dewan, Irin. "Comparative in vitro dissolution study of Aceclofenac Marketed Tablets in Two Different Dissolution Media by Validated Analytical Method." (2011).

15. Song, Nai-Ning \& Zhang, Shao-Yu \& Liu, Chang-Xiao. (2004). Overview of factors affecting oral drug absorption.Asian J Drug Metabolism Pharmacokinetics. 4. 167-176.

16. Buxton ILO. (2006) In - Goodman \& Gilman's - The Pharmacological basis of Therapeutics $11^{\text {th }}$ Edition, McGraw-Hill Medical Publishing Division, Digital Edition, Chapter 1.

17. Augsburger L. L, Shangraw R. F, Giannini R. P, Shah V. P, Prasad V. K, Brown D. (1983) Thiazides VIII: Dissolution Survey of marketed Hydrochlorothiazide tablets. J. Pharm. Sci., 72(8): 876-881.

18. USP General Chapter on Dissolution . United States Pharmacopeia and National Formulary; United States Pharmacopoeial Convention, Inc.: Rockville, MD, 2005; pp 2412-2414.

19. Levy, G. Effect of dissolution rate on absorption, metabolism, and pharmacologic activity of drugs. J. Mond. Pharm. 1967, 3, 237-254.

20.Wagner, J. G. Biopharmaceutics and Relevant Pharmacokinetics; Drug Intelligence Publications: Hamilton, IL, 1971; Chapter 10.

21. Waiver of In Vivo Bioavailability and Bioequivalence Studies for Immediate Release Solid Oral Forms Containing Certain Active Moieties/Active Ingredients Based on Biopharmaceutics Classification System; Guidance 
for Industry; U.S. Department of Health and Human Services, Food and Drug Administration, Center for Drug Evaluation and Research (CDER), U.S. Government Printing Office: Washington, DC, 2000.

http://www.fda.gov/cder/guidance/3618fnl.pdf

22. Setty C. M, Prasad D. V, Gupta V. R, Sa B. Development of fast dispersible aceclofenac tablets: effect of functionality of superdisintegrants. Indian J Pharm Sci. 2008;70(2):180-5.

23. https://www.spipharma.com/media/2381/effer-soda-product-sales-bulletin.pdf

24. Srivastav M., Prabhakar B., Omray A. Effect of alkaline excipients on the release profile of gliclazide extended release tablets. J. Pharm. Sci. Technol. 2011;3(1):462-469. 